Tôhoku Math. Journ.

28 (1976), 601-612.

\title{
CURVATURE IDENTITIES FOR HERMITIAN AND ALMOST HERMITIAN MANIFOLDS
}

\author{
Alfred Gray
}

(Received September 25, 1975)

1. Introduction. Among Riemannian manifolds Kähler manifolds have an especially rich geometric and topological structure. Perhaps the main reason for this is that the curvature tensor of a Kähler manifold satisfies a special identity, the Kähler identity $R_{W X J Y J Z}=R_{W X Y Z}$. From this identity it is possible to prove many interesting theorems about harmonic forms, for example.

Because of the strength of the Kähler identity, the question naturally arises: what sort of identities, if any, exist for more general types of almost Hermitian manifolds. In this paper we show that indeed curvature identities exist for the classes $\mathscr{C}$ and $\mathscr{Q} \mathscr{K}$ of Hermitian and quasiKähler manifolds.

That a curvature identity for Hermitian manifolds has not been found or made use of up to now in the literature is somewhat surprising. The defining property of the class $\mathscr{C}$ is that

$$
[X, Y]+J[J X, Y]+J[X, J Y]-[J X, J Y]=0 .
$$

We shall show that

$$
\left[R_{X Y}, J\right]+J\left[R_{J X Y}, J\right]+J\left[R_{X J Y}, J\right]-\left[R_{J X J Y}, J\right]=0
$$

holds for $\mathscr{H}$. Since the structure of Hermitian manifolds is almost as rich as that of Kähler manifolds, this identity should prove productive.

Quasi-Kähler manifolds are important because they include the classes $\mathscr{A} \mathscr{K}$ and $\mathscr{N} \mathscr{K}$ of almost Kähler and nearly Kähler manifolds. A large part of the theory of the geometry and topology of Kähler manifolds can be carried over to the class $\mathscr{N} \mathscr{K}$ [8]. However, not very much is known about Aצ\%. We give a new curvature identity in Corollary (4.3). Hervella and Naveira [12] have derived a special case of our curvature identity for the class $\mathbb{Q} \mathscr{K}$. They also give several geometric applications.

In $\S 2$ we explain our notation and prove an important lemma. We study curvature identities of Hermitian manifolds in $\S 3$. Then $\S 4$ is devoted to curvature identities in quasi-Kähler and almost Kähler mani- 
folds. In $\S 5$ we give a partial answer to the question: if the curvature tensor of an almost Hermitian manifold $M$ is similar to that of a Kähler manifold, must $M$ be Kählerian? In $\S 6$ we consider manifolds with an almost complex structure defined by a vector cross product, and apply the results of the preceding sections.

$\S 7$ is devoted to a geometrical application of the curvature identities. We give general conditions under which the distribution $m \rightarrow \mathscr{K}(m)=$ $\left\{x \in M_{m} \mid \nabla_{x}(J) y=0\right.$ for all $\left.y \in M_{m}\right\}$ is an integrable distribution.

In $\S 8$ we give a sufficient condition in terms of the holomorphic sectional curvature for any complete almost Hermitian manifold to be compact and simply connected.

I wish to thank L. M. Hervella, A. M. Naveira and L. Vanhecke for several useful conversations.

2. A curvature identity. Let $M$ be a $C^{\infty}$ almost Hermitian manifold with metric $\langle$,$\rangle and almost complex structure J$. Denote by $\mathscr{Q}(M)$ the Lie algebra of $C^{\infty}$ vector fields on $M$. The Nijenhuis tensor $S$ of $J$ is defined by

$$
S(X, Y)=[X, Y]+J[J X, Y]+J[X, J Y]-[J X, J Y],
$$

for $X, Y \in \mathscr{X}(M)$. A well-known theorem [2], [14] states that $M$ is a complex manifold if and only if $S$ vanishes identically.

Let $\nabla_{X}, R_{X Y}(X, Y \in \mathscr{X}(M))$ denote the covariant derivative and curvature operator of $M$. We now prove a curvature identity which will be useful for both Hermitian and quasi-Kähler manifolds. It will be convenient to use the notation $\left[\nabla_{X}, J\right]=\nabla_{X} J-J \nabla_{X}$.

LEMMA 2.1. Let $\varepsilon= \pm 1$, and assume that $M$ has the property that $\left[\nabla_{J X}, J\right]=\varepsilon J\left[\nabla_{X}, J\right]$ for all $X \in \mathscr{Z}(M)$. Then

$$
\left[\nabla_{S(X, Y)}, J\right]=\left[R_{X Y}-R_{J X J Y}, J\right]+\varepsilon J\left[R_{J X Y}+R_{X J Y}, J\right]
$$

for $X, Y \in \mathscr{Q}(M)$.

Proof. The curvature operator $R_{X Y}(X, Y \in \mathscr{P}(M))$ is defined by

$$
R_{X Y}=\nabla_{[X, Y]}-\left[\nabla_{X}, \nabla_{Y}\right] \text {. }
$$

From (2.1) and (2.2) it follows that

$$
\begin{aligned}
& {\left[\nabla_{S(X, Y)}, J\right]-\left[R_{X Y}-R_{J X J Y}, J\right]-\varepsilon J\left[R_{J X Y}+R_{X J Y}, J\right]} \\
& \quad=\left[\left[\nabla_{X}, \nabla_{Y}\right]-\left[\nabla_{J X}, \nabla_{J Y}\right], J\right]+\varepsilon J\left[\left[\nabla_{J X}, \nabla_{Y}\right]+\left[\nabla_{X}, \nabla_{J Y}\right], J\right] .
\end{aligned}
$$

We use the Jacobi identity on each of the terms on the right hand side of (2.3), and then we use $\left[\nabla_{J X}, J\right]=\varepsilon J\left[\nabla_{X}, J\right]$. It follows that the right 
hand side of (2.3) vanishes, and so we obtain the lemma.

3. Hermitian manifolds. As an immediate consequence of Lemma 2.1 we obtain a curvature identity for the class $\mathscr{H}$ of Hermitian manifolds. This identity formally resembles (2.1).

Theorem 3.1. For $M \in \mathscr{H}$ and $X, Y \in \mathscr{X}(M)$ we have

$$
\left[R_{X Y}, J\right]+J\left[R_{J X Y}, J\right]+J\left[R_{X J Y}, J\right]-\left[R_{J X J Y}, J\right]=0 .
$$

Proof. In [4], [5] it is shown that $M \in \mathscr{H}$ if and only if $\left[\nabla_{J X}, J\right]=$ $J\left[\nabla_{X}, J\right]$ for $X \in \mathscr{Q}(M)$, and this is true if and only if $S=0$. Thus we take $\varepsilon=1$ in Lemma 2.1 and obtain (3.1).

We write $R_{W X Y Z}=\left\langle R_{W X} Y, Z\right\rangle$ for $W, X, Y, Z \in \mathscr{X}(M)$. The sectional curvature of $M$ is given by $K_{W X}=R_{W X W X}\left\{\|W\|^{2}\|X\|^{2}-\langle W, X\rangle^{2}\right\}^{-1}$. The following corollary is immediate from Theorem 3.1.

Corollary 3.2. Let $M \in \mathscr{H}$. Then

$$
\begin{aligned}
R_{W X Y Z}+R_{J W J X J Y J Z} & -R_{J W J X Y Z}-R_{J W X J Y Z}-R_{J W X Y J Z} \\
& -R_{W J X J Y Z}-R_{W J X Y J Z}-R_{W X J Y J Z}=0
\end{aligned}
$$

for $W, X, Y, Z \in \mathscr{Q}(M)$.

$$
K_{W X}+K_{J W J X}-K_{W J X}-K_{J W X}=2 R_{W X J W J X}+2 R_{W J X J W X}
$$

when $\|W\|=\|X\|=1$ and $\langle W, X\rangle=0$.

Also, we have

COROLlaRy 3.3. Let $M \in \mathscr{H}$. Then the following two curvature conditions are equivalent

$$
R_{W X Y Z}=R_{J W J X J Y Z} \text { for all } W, X, Y, Z \in \mathscr{Z}(M),
$$

The following are three types of non-Kähler Hermitian manifolds which have particularly simple curvature.

(1) Compact homogeneous complex manifolds. These have been classified [17] and include compact even-dimensional Lie groups and the products of the spheres $S^{2 n+1}\left(r_{1}\right) \times S^{2 m+1}\left(r_{2}\right)$.

(2) Manifolds conformally equivalent to Kähler manifolds, in particular $\boldsymbol{C}^{n}$.

(3) Certain 6-dimensional orientable submanifolds of $\boldsymbol{R}^{8}$. These are defined by means of a 3 -fold vector cross product on $\boldsymbol{R}^{8}$.

The curvature operator of a compact homogeneous complex manifold in general does not satisfy any identity simpler than (3.1). 
In [10] Hermitian manifolds conformally equivalent to $\boldsymbol{C}^{n}$ are considered. In particular new examples of manifolds with constant holomorphic sectional curvature $\mu$ are given. It is shown that such manifolds satisfy

$$
\begin{aligned}
R_{W X W X}+ & R_{J W J X J W J X}-R_{W J X W J X}-R_{J W X J W X} \\
& =2 \mu\left\{-\langle W, X\rangle^{2}+\langle J W, X\rangle^{2}\right\}, \quad W, X \in \mathscr{C}(M) .
\end{aligned}
$$

Six dimensional orientable submanifolds of $\boldsymbol{R}^{8}$ are discussed in $\S 6$.

4. Quasi-Kähler manifolds. Next we apply Lemma 2.1 to the class Q. $\mathscr{K}$ of quasi-Kähler manifolds.

THEOREM 4.1. For $M \in \mathscr{Q} \mathscr{K}$ and $X, Y \in \mathscr{X}(M)$ we have

$$
\begin{aligned}
{\left[R_{X Y}-R_{J X J Y}, J\right]-J\left[R_{J X Y}+R_{X J Y}, J\right] } \\
=2 J\left[\nabla_{\left[\nabla_{X}, J\right] Y}, J\right]-2 J\left[\nabla_{\left[7_{Y}, J\right] X}, J\right] .
\end{aligned}
$$

Proof. By definition $M \in \mathscr{Q} \mathscr{K}$ if and only if $\left[\nabla_{J X}, J\right]=-J\left[\nabla_{X}, J\right]$ for $X \in \mathscr{P}(M)$. Furthermore an easy calculation shows $S(X, Y)=$ $2 J\left\{\left[\nabla_{X}, J\right] Y-\left[\nabla_{Y}, J\right] X\right\}$ for $X, Y \in \mathscr{P}(M)$. Thus we obtain (4.1) from Lemma 2.1.

Corollary 4.2. Let $M \in \mathscr{Q} \mathscr{K}$. Then

$$
\begin{aligned}
R_{W X Y Z} & +R_{J W J X J Y J Z}-R_{J W J X Y Z}-R_{W X J Y J Z} \\
& +R_{J W X J Y Z}+R_{W J X Y J Z}+R_{J W X Y J Z}+R_{W J X J Y Z} \\
= & 2\left\langle\left[\nabla_{\left[\nabla_{W}, J\right] X}, J\right] Y, Z\right\rangle+2\left\langle\left[\nabla_{\left[\nabla_{X}, J\right] W}, J\right] Z, Y\right\rangle
\end{aligned}
$$

for $W, X, Y, Z \in \mathscr{X}(M)$;

$$
\begin{aligned}
& K_{W X}+K_{J W J X}+K_{W J X}+K_{J W X}-2 R_{W J W X J X} \\
& =2\left\langle\left[\nabla_{\left[\nabla_{W}, J\right] X}, J\right] W, X\right\rangle+2\left\langle\left[\nabla_{\left[\nabla_{X}, J\right] W}, J\right] X, W\right\rangle
\end{aligned}
$$

when $\|W\|=\|X\|=1$ and $\langle W, X\rangle=0$.

The class $\mathscr{Q} \mathscr{K}$ contains two important subclasses $\mathscr{N} \mathscr{K}$ and $\mathscr{A} \mathscr{K}$. Curvature identities and their consequences for the class $\mathscr{N} \mathscr{K}$ of nearly Kähler manifolds are discussed extensively in [8], [9], and [13]. Theorem (4.1) yields no new information for $\mathscr{N} \mathscr{K}$.

On the other hand, for the class $\mathscr{A} \mathscr{K}$ of almost Kähler manifolds we have the following new identity.

Corollary 4.3. Let $M \in \mathscr{A} \mathscr{K}$. Then

$$
\begin{aligned}
R_{W X Y Z} & +R_{J W J X J Y J Z}+R_{J W X J Y Z}+R_{W J X Y J Z} \\
& -R_{J W J X Y Z}-R_{W X J Y J Z}+R_{J W X Y J Z}+R_{W J X J Y Z} \\
= & -2\left\langle\left[\nabla_{W}, J\right] X-\left[\nabla_{X}, J\right] W,\left[\nabla_{Y}, J\right] Z-\left[\nabla_{Z}, J\right] Y\right\rangle,
\end{aligned}
$$


for $W, X, Y, Z \in \mathscr{Z}(M)$.

(4.5) $K_{W X}+K_{J W J X}+K_{W J X}+K_{J W X}-2 R_{W J W X J X}=-2\left\|\left[\nabla_{W}, J\right] X-\left[\nabla_{X}, J\right] W\right\|^{2}$ when $\|W\|=\|X\|=1,\langle W, X\rangle=0$.

Proof. The Kähler form $F$ on an almost Hermitian manifold $M$ is defined by $F(X, Y)=\langle J X, Y\rangle$ for $X, Y \in \mathscr{X}(M)$. By definition $M \in$ $\mathscr{A} \mathscr{C}$ if and only if $d F=0$. This is equivalent to

$$
\left\langle\left[\nabla_{W}, J\right] X, Y\right\rangle+\left\langle\left[\nabla_{Y}, J\right] W, X\right\rangle+\left\langle\left[\nabla_{X}, J\right] Y, W\right\rangle=0
$$

for $W, X, Y \in \mathscr{Z}(M)$. Then (4.4) and (4.5) follow from (4.6) and (4.2).

5. Almost Hermitian manifolds whose curvature operator resembles that of a Kähler manifold. Consider the following identities for the curvature operator of an almost Hermitian manifold $M$ :

(1) $R_{W X Y Z}=R_{W X J Y J Z}$,

(2) $R_{W X Y Z}-R_{J W J X Y Z}=R_{J W X J Y Z}+R_{J W X Y J Z}$,

(3) $R_{W X Y Z}=R_{J W J X J Y J Z}, \quad W, X, Y, Z \in \mathscr{Q}(M)$.

For a given class $\mathscr{L}$ of almost Hermitian manifolds, let $\mathscr{L}_{i}$ be the subclass of manifolds whose curvature operator satisfies identity (i). It is easily shown that $\mathscr{L}_{1} \subseteq \mathscr{L}_{2} \subseteq \mathscr{L}_{3} \subseteq \mathscr{L}$. Thus as $i$ decreases the manifolds in $\mathscr{L}_{i}$ resemble Kähler manifolds more closely.

We remark parenthetically that the identities (1), (2), (3) can be considered from the standpoint of group representations. For each $m \in M$ there is a representation of the unitary group $U(n)$ on the space of curvature tensors at $m, \mathscr{R}\left(M_{m}\right)$. This representation is always reducible. In fact each of the identities is the defining equation for certain sums of the components of the representation. One cannot obtain all components in this way, but the identities (1), (2), (3) are the most natural.

Certain equalities occur among the various classes. We summarize the known results.

THEOREM 5.1. We have

$$
\begin{aligned}
\mathscr{K}_{1} & =\mathscr{K}_{2}=\mathscr{K}_{3}=\mathscr{K} ; \\
\mathscr{K} & =\mathscr{N} \mathscr{K}_{1} ; \\
\mathscr{N} \mathscr{K}_{2} & =\mathscr{N} \mathscr{K}_{3}=\mathscr{N} \mathscr{K} ; \\
\mathscr{K} & =\mathscr{H} \mathscr{K}_{1} ; \\
\mathscr{K}_{2} & =\mathscr{H}_{3} .
\end{aligned}
$$

Proof. (5.1) is well-known, and (5.2), (5.3), (5.4) are proved in [6]. Although each of these is a local theorem, a global proof of (5.4) is 
given in [11]. The proof [11] although less general is nonetheless quite interesting. (5.2) and (5.4) are also proved in [15]. However, we see now that (5.4) is a simple consequence of Corollary 4.3.

Finally (5.5) is a consequence of Corollary 3.3.

We have not been able to solve all of the inclusion relations involving the identities (1), (2), (3). However, the following theorem gives nearly complete results. We use the notation " $\subset$ " to indicate that a given inclusion is strict and "œ" to indicate that we don't know.

THEOREM 5.3. We have

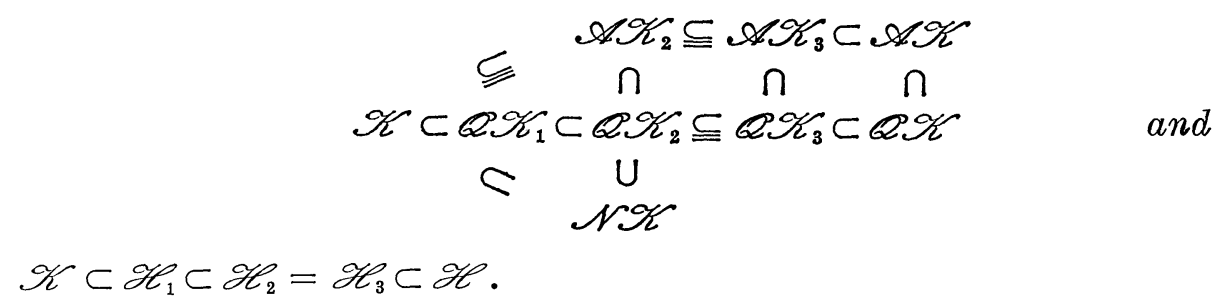

PRoof. That all the inclusions exist is proved in [4], [5], [13]. Therefore it remains to give an example for each strict inclusion.

In [9] certain 3-symmetric spaces $M$ are described. It is shown that $M \in \mathbb{Q} \mathscr{K}_{2}$, but $M \notin \mathscr{N} \mathscr{K}$ and $M \notin \mathscr{A} \mathscr{K}$. Thus we have $\mathscr{N} \mathscr{K} \subset \mathbb{Q} \mathscr{K}_{2}$, $\mathscr{A} \mathscr{K}_{2} \subset \mathbb{Q} \mathscr{K}_{2}, \mathscr{A} \mathscr{K}_{3} \subset \mathbb{Q} \mathscr{K}_{3}$, and $\mathscr{A} \mathscr{K} \subset \mathbb{Q} \mathscr{K}_{\text {. Also }} Q \mathscr{K}_{1} \subset \mathbb{Q} \mathscr{K}_{2}$.

It is shown in [6] that $S^{2} \times R^{4}$ has a quasi-Kähler almost complex structure $J$ induced from a vector cross product on $\boldsymbol{R}^{7}$. One can check that the curvature operator of $S^{2} \times \boldsymbol{R}^{4}$ has the interesting property that

$$
R_{W X Y Z}=R_{W X J Y J Z} \quad \text { for } \quad W, X, Y, Z \in \mathscr{Q}\left(S^{2} \times R^{4}\right) \text {. }
$$

(See also Theorem 6.6.) Thus we have $\mathscr{K} \subset \mathbb{Q} \mathscr{K}_{1}$.

If $M$ is any Riemannian manifold then the tangent bundle $T(M)$ is almost Kählerian [1]. If $M$ is not flat and $\operatorname{dim} M \geqq 2$ one can check that $T(M) \notin \mathscr{A} \mathscr{K}_{3}$. Thus $\mathscr{A} \mathscr{K}_{3} \subset \mathscr{A} \mathscr{K}$ and $\mathscr{Q} \mathscr{K}_{3} \subset \mathbb{Q} \mathscr{K}$. The product $S^{2 n+1}\left(r_{1}\right) \times S^{2 m+1}\left(r_{2}\right)(n, m>0)$ is Hermitian but not in $\mathscr{H}_{3}$. Hence $\mathscr{H}_{3} \subset \mathscr{H}$.

In $\S 6$ we shall prove that $\mathscr{K} \subset \mathscr{H}_{1}$ by exhibiting a certain 6dimensional Hermitian manifold. To prove that $\mathscr{H}_{1} \subset \mathscr{H}_{2}$ we observe that any contractible Riemannian manifold $M$ of nonzero constant sectional curvature has an integrable almost complex structure. If dim $M \geqq 4$, then $M \in \mathscr{H}_{2}$ but $M \notin \mathscr{H}_{1}$. From (5.5) we have $\mathscr{H}_{2}=\mathscr{H}_{3}$.

6. Almost complex manifolds defined by means of a 3 -fold vector cross product. In [6] it is shown that every orientable 6-dimensional 
submanifold $M$ of $\boldsymbol{R}^{8}$ has an almost complex structure induced from a 3-fold vector cross product on $\boldsymbol{R}^{8}$. Since there are two different ways to induce a vector cross product on $\boldsymbol{R}^{8}, M$ has (in general) two different almost complex structures. In this section we show that Hermitian and quasi-Kähler manifolds constructed in this way have some remarkable curvature properties.

Let $P$ denote a 3 -fold vector cross product on $\boldsymbol{R}^{8}$. We consider $P$ as a tensor field of type (3.1). If $M \subset R^{8}$ is orientable, the normal bundle of $M$ has an almost complex structure $J$ defined on it. Let $N$ be a unit normal on an open subset of $M$. Then $N \wedge J N$ is independent of $N$, and we may extend it to a global 2-field on $M$. The almost complex structure $J$ on $M$ is well-defined by the equation $J X=P(N, J N, X)$ for $X \in \mathscr{P}(M)$.

Throughout the rest of this section we shall assume that $M$ has an almost complex structure given by $J X=P(N, J N, X)$.

TheOREM 6.1. Suppose $M \in \mathscr{H}$. Then

$$
R_{W X Y Z}-R_{W X J Y J Z}=0 \text { for } W, X, Y, Z \in \mathscr{Z}(M) \text {. }
$$

Proof. In [6] it is shown that

$$
T_{W} X+T_{J W} J X=0 \text { for } W, X \in \mathscr{X}(M),
$$

where $T$ denotes the configuration tensor of $M$ in $\boldsymbol{R}^{8}$ [4]. The curvature operator of $M$ satisfies the Gauss equation

$$
R_{W X Y Z}=\left\langle T_{W} Y, T_{X} Z\right\rangle-\left\langle T_{W} Z, T_{X} Y\right\rangle \text {. }
$$

From (6.2) and (6.3) it follows easily that

$$
R_{W X Y Z}-R_{W X J Y J Z}+R_{W J X Y J Z}+R_{J W J X J Y J Z}=0,
$$

and

$$
R_{W X Y Z}=R_{J W J X J Y J Z} .
$$

Now (6.1) follows from (6.4), (6.5), and (3.5).

In [3], [6] 6-dimensional Hermitian manifolds are constructed in the following way.

THEOREM 6.2. Let $V \subset \boldsymbol{R}^{8}$ be any 4-dimensional linear subspace closed under the vector cross product $P$. Let $M^{*} \subset V$ be any 2-dimensional orientable submanifold, and form the product $M^{*} \times V^{\perp}$. Then $M^{*} \times V^{\perp} \in \mathscr{H}$ if and only if $M^{*}$ is a minimal variety of $V$. In particular if $M^{*}$ is contained in a hypersurface of $V$, then $M^{*} \times V^{\perp} \epsilon$ $\mathscr{H}$ if and only if $M^{*}$ is an ordinary minimal surface in 3-space.

We now prove a partial converse to this theorem. 
THEOREM 6.3. Let $M$ be 6-dimensional orientable submanifold of a 7-dimensional linear subspace $\boldsymbol{R}^{7}$ of $\boldsymbol{R}^{8}$, and assume $M \in \mathscr{H}$. Then $M$ has at most two nonzero principal curvatures $\kappa_{1}$ and $\kappa_{2}$; moreover $\kappa_{1}=-\kappa_{2}$. If $\kappa_{1}$ and $\kappa_{2}$ are nonzero, the eigenspaces corresponding to them have dimension 2.

Proof. From (6.1) and (6.3) we have (6.6) $\left\langle T_{W} W, T_{X} X\right\rangle-\left\langle T_{W} J W, T_{X} J X\right\rangle-\left\|T_{W} X\right\|^{2}+\left\|T_{W} J X\right\|^{2}=0$ for $W, X \in \mathscr{Z}(M)$. Now assume

$$
T_{W} N=\kappa_{1} W, \quad T_{X} N=\kappa_{2} X,
$$

$N$ is the unit normal to $M$ in $\boldsymbol{R}^{7}$. From (6.6) we obtain

$$
\kappa_{1} \kappa_{2}\|W\|^{2}\|X\|^{2}-\kappa_{1}^{2}\langle W, X\rangle^{2}+\kappa_{1}^{2}\langle W, J X\rangle^{2}=0 .
$$

Hence if $\langle W, X\rangle=\langle W, J X\rangle=0,\|W\|=\|X\|=1$, then $\kappa_{1} \kappa_{2}=0$. It is easy to check that $T_{J W} N=-\kappa_{1} J W$ etc. Hence the theorem follows.

COROLLARY 6.4. Let $M$ satisfy the hypotheses of Theorem 6.3. Then $M=M^{*} \times \boldsymbol{R}^{4}$ where $M^{*}$ is an ordinary minimal surface contained in a 3-dimensional linear subspace.

Now we turn to the class QY\%.

THEOREM 6.5. Suppose $M \in \mathbb{Q} \mathscr{C}$. Then

$$
\begin{aligned}
& R_{W X Y Z}+R_{J W J X J Y J Z}-R_{J W J X Y Z}-R_{W X J Y J Z} \\
& =\left\langle\left[\nabla_{\left[\nabla_{W}, J\right] X}, J\right] Y, Z\right\rangle+\left\langle\left[\nabla_{\left.{ }^{[}{ }_{X}, J\right] W}, J\right] Z, Y\right\rangle
\end{aligned}
$$

for $W, X, Y, Z \in \mathscr{Z}(M)$.

Proof. In [6] it is shown that

$$
T_{W} X-T_{J W} J X= \pm J\left(T_{J W} Y+T_{W} J Y\right)
$$

for $W, X \in \mathscr{Z}(M)$. Here "+" or "- " is chosen in (6.9) depending on which of the two vector cross products on $\boldsymbol{R}^{8}$ is used to define the almost complex structure on $M$.

From (6.9) and (6.3) after some calculation we obtain

$$
\begin{aligned}
R_{W X Y Z} & +R_{J W J X Y Y Z}-R_{J W J X Y Z}-R_{W X J Y J Z} \\
& -R_{W J X Y J Z}-R_{J W X J Y Z}-R_{W J X J Y Z}-R_{J W X Y J Z}=0 .
\end{aligned}
$$

Now (6.8) follows from (6.10) and (4.2).

We can also consider quasi-Kähler manifolds contained in a hyperplane of $\boldsymbol{R}^{8}$. From Theorem 6.5 it is not difficult to show that such a manifold $M$ can have at most two distinct principal curvatures. In fact in 
[6] it is shown that $M$ is an open subset of $R^{6}, S^{6}$ or $S^{2} \times R^{4}$. From Theorem 6.5 we see that these manifolds satisfy simple curvature identities.

THEOREM 6.6. Let $M \in \mathscr{Q} \mathscr{K}$ and assume $M$ is contained in a hyperplane $\boldsymbol{R}^{7} \subset \boldsymbol{R}^{8}$. (In other words $M$ is a piece of $\boldsymbol{R}^{6}, S^{6}$, or $S^{2} \times \boldsymbol{R}^{4}$, imbedded isometrically in an appropriate fashion). Then

$$
R_{W X Y Z}=R_{J W J X Y J Z}
$$

$$
R_{W X Y Z}-R_{W X J Y J Z}=\frac{1}{2}\left\{\left\langle\left[\nabla_{\left[\nabla_{W}, J\right] X}, J\right] Y, Z\right\rangle+\left\langle\left[\nabla_{\left[\nabla_{X}, J\right] W}, J\right] Z, Y\right\rangle\right\} ;
$$

where $W, X, Y, Z \in \mathscr{P}(M)$.

Proof. Since $M$ is contained in a hyperplane equation (6.9) reduces to

$$
T_{W} X=T_{J W} J X \text { for } W, X \in \mathscr{P}(M) \text {. }
$$

Then (6.11) follows easily from (6.13) and (6.3). We then obtain (6.12) from (6.11) and (6.8).

In fact $S^{6}$ satisfies the identity

$$
R_{W X Y Z}-R_{W X J Y J Z}=\left\langle\left[\nabla_{W}, J\right] X,\left[\nabla_{Y}, J\right] Z\right\rangle,
$$

while $S^{2} \times R^{4}$ satisfies

$$
R_{W X Y Z}-R_{W X J Y J Z}=0 \text {. }
$$

Finally, we consider the class $\mathscr{4} \mathscr{K}$. It is proved in [6] that if $M \in \mathscr{A} \mathscr{K}$ then $M$ is a minimal variety of $\boldsymbol{R}^{8}$, and cannot be compact. Furthermore if $M$ is contained in a hyperplane, $M$ must be a piece of $\boldsymbol{R}^{6}$.

For general $M \in \mathscr{C} \mathscr{C}$ we have the following consequence of Theorem 6.5 .

Corollary 6.7. Suppose $M \in \mathscr{A} \mathscr{K}, M \subset \boldsymbol{R}^{8}$. Then

$$
\begin{aligned}
& R_{W X Y Z}+R_{J W J X J Y J Z}-R_{J W J X Y Z}-R_{W X J Y J Z} \\
& \quad=-\left\langle\left[\nabla_{W}, J\right] X-\left[\nabla_{X}, J\right] W,\left[\nabla_{Y}, J\right] Z-\left[\nabla_{Z}, J\right] Y\right\rangle .
\end{aligned}
$$

\section{Integrability of spaces of Kähler-nullity.}

Definition. Let $M$ be an almost Hermitian manifold. For each $m \in M$ we set

$$
\mathscr{K}(m)=\left\{x \in M_{m} \mid \nabla_{X}(J) y=0 \text { for all } y \in M_{m}\right\} .
$$

We call $\mathscr{K}(m)$ the space of Kähler nullity.

In [8] it was proved that $m \rightarrow \mathscr{K}(m)$ is integrable provided $M$ is a nearly Kähler manifold. The same result holds when $M$ is a quasiKähler manifold satisfying curvature identity (1). This is proved in 
[12]. The following theorem generalizes both of these results.

THEOREM 7.1. Let $M \in \mathbb{Q} \mathscr{K}$ satisfy the curvature identity (2), namely $R_{W X Y Z}-R_{W X J Y J Z}=R_{W J X Y J Z}+R_{J W X Y J Z}$ for $W, X, Y, Z \in \mathscr{X}(M)$. Then on any open subset of $M$ on which $\operatorname{dim} \mathscr{K}(m)$ is constant, the distribution $m \rightarrow \mathscr{K}(m)$ is integrable. Furthermore any integral manifold of $m \rightarrow \mathscr{K}(m)$ is a Kähler submanifold of $M$.

Proof. From Corollary 4.2 it follows that

$$
R_{W X Y Z}-R_{W X J Y J Z}=\frac{1}{2}\left\langle\left[\nabla_{\left[\nabla_{W}, J\right] X}, J\right] Y, Z\right\rangle+\frac{1}{2}\left\langle\left[\nabla_{\left[\nabla_{X}, J\right] W}, J\right] Z, Y\right\rangle
$$

for $W, X, Y, Z \in \mathscr{X}(M)$. Now let $W, X$ be vector fields which at each point lie in the distribution $m \rightarrow \mathscr{K}(m)$. Then for $Y, Z \in \mathscr{X}(M)$ we have

$$
\left\langle\left[\nabla_{[W, X]}, J\right] Y, J Z\right\rangle=\left\langle\left(\left[R_{W X}, J\right]+\left[\left[\nabla_{W}, \nabla_{X}\right], J\right]\right) Y, J Z\right\rangle \cdot
$$

We use the Jacobi identity on $\left[\left[\nabla_{W}, \nabla_{X}\right], J\right]$. Thus (7.2) reduces to

$$
\left\langle\left[\nabla_{[W, X]}, J\right] Y, J Z\right\rangle=R_{W X J Y J Z}-R_{W X Y Z} \cdot
$$

In view of (7.1), the right hand side of (7.3) vanishes. Hence $[W, X]$ lies in the distribution $m \rightarrow \mathscr{K}(m)$. Therefore, by the Frobenius theorem, it follows that $m \rightarrow \mathscr{K}(m)$ is integrable on open sets of $M$ on which $\operatorname{dim} \mathscr{K}(m)$ is constant.

Let $M^{\prime}$ be an integral submanifold of $m \rightarrow \mathscr{K}(m)$. That $M^{\prime}$ is a Kähler submanifold of $M$ is proved in exactly the same way as in [8].

We also have the corresponding theorem valid for any almost Hermitian manifold $M$.

THEOREM 7.2. Let $M$ satisfy the curvature identity (1), namely $R_{W X Y Z}-R_{W X J Y J Z}=0$ for $W, X, Y, Z \in \mathscr{P}(M)$. Then on any open subset of $M$ on which $\operatorname{dim} \mathscr{K}(m)$ is constant, the distribution $m \rightarrow \mathscr{K}(m)$ is integrable. Furthermore any integral manifold of $m \rightarrow \mathscr{K}(m)$ is a Kähler submanifold of $M$.

We omit the proof which is very similar to that of Theorem 7.3.

8. Compactness of almost Hermitian manifolds. Tsukamoto [16] has proved that a complete Kähler manifold must be compact if its holomorphic sectional curvature is bounded below by a positive constant. In fact, the same theorem is true for nearly Kähler manifolds [8]. In this section we present a simple generalization of these results, valid for any almost Hermitian manifold. 
THEOREM 8.1. Let $M$ be a complete almost Hermitian manifold. Assume that the holomorphic sectional curvature of $M$ satisfies

$$
K_{x J x}-\left\|\nabla_{x}(J) x\right\|^{2}\|x\|^{-4} \geqq \delta>0
$$

for all $x \in M_{m}$ and all $m \in M$. Then $M$ is compact and the diameter of $M$ is not greater than $\pi / \sqrt{\delta}$.

Proof. Let $p, q \in M$. Since $M$ is complete, there exists a unique unit speed geodesic $\sigma$ defined on $[0, b]$ from $p$ to $q$. Let $X$ be the vector field on $\sigma$ defined by $X(t)=\sin (\pi t / b) J \sigma^{\prime}(t)$. The deformation of $\sigma$ given by $X$ has second variation

$$
\begin{aligned}
I(X, X) & =\int_{0}^{b}\left\{\left\|X^{\prime}\right\|^{2}-\left\langle R_{X \sigma^{\prime}} X, \sigma^{\prime}\right\rangle\right\}(t) d t \\
& =\int_{0}^{b}\left\{\frac{\pi^{2}}{b^{2}} \cos ^{2} \frac{\pi t}{b}+\sin ^{2} \frac{\pi t}{b}\left(\left\|\nabla_{\sigma^{\prime}}(J) \sigma^{\prime}\right\|^{2}-K_{\sigma^{\prime} J \sigma^{\prime}}\right)\right\} d t \\
& \leqq \int_{0}^{b}\left\{\frac{\pi^{2}}{b^{2}} \cos ^{2} \frac{\pi t}{b}-\delta \sin ^{2} \frac{\pi t}{b}\right\} d t \\
& \leqq \frac{b}{2}\left(\frac{\pi^{2}}{b^{2}}-\delta\right) .
\end{aligned}
$$

Hence, if $b>\pi / \sqrt{\delta}$, then $I(X, X)<0$, and so $\sigma$ has a conjugate point. Since any two points are connected by a unique minimal geodesic, the theorem follows.

It follows from Theorem 8.1 that a complete almost Hermitian manifold satisfying (8.1) must have finite fundamental group. In fact, the following result is true.

THEOREM 8.2. Let $M$ be a complete almost Hermitian manifold whose holomorphic sectional curvature satisfies (8.1). Then $M$ is simply connected.

Proof. Assume the contrary. Then there exists a non-trivial free homotopy class of loops which contains a non-trivial minimal geodesic $\sigma$. We may assume that $\sigma$ has unit speed and is defined on $[0, b]$. The deformation of $\sigma$ given by $J \sigma^{\prime}$ has second variation

$$
I\left(J \sigma^{\prime}, J \sigma^{\prime}\right)=\int_{0}^{b}\left\{\left\|\nabla_{\sigma^{\prime}}(J) \sigma^{\prime}\right\|^{2}-K_{\sigma^{\prime} J \sigma^{\prime}}\right\}(t) d t .
$$

Thus by (8.1), $I\left(J \sigma^{\prime}, J \sigma^{\prime}\right)<0$, and so $\sigma$ cannot be a minimal geodesic. Hence $M$ is simply connected. 


\section{REFERENCES}

[1] P. Dombrowski, On the geometry of tangent bundles, J. Reine Angew. Math., 210 (1962), 73-88.

[2] A. Frohlicher, Zur Differentialgeometrie der komplexen Strukturen, Math. Ann., 129 (1955), 50-95.

[3] E. CALABI, Construction and properties of some 6-dimensional almost complex manifolds, Trans. Amer. Math. Soc., 87 (1958), 407-438.

[4] A. Gray, Minimal varieties and Hermitian submanifolds, Michigan Math. J., 12 (1965), 273-287.

[5] A. Gray, Some examples of almost Hermitian manifolds, Illinois J. Math., 10 (1966), 353-366.

[6] A. Gray, Vector cross products on manifolds, Trans. Amer. Math. Soc., 141 (1969), 465-504.

[7] A. Gray, Six dimensional almost complex manifolds defined by means of three fold vector cross products, Tôhoku Math. J., 21 (1969), 614-620.

[8] A. GraY, Nearly Kähler manifolds, J. of Differential Geometry, 4 (1970), 283-309.

[9] A. GraY, Riemannian manifolds with geodesic symmetries of order 3, J. Differential Geometry, 7 (1972), 343-369.

[10] A. Gray and L. Vanhecke, Almost Hermitian manifolds with constant holomorphic sectional curvature, (to appear).

[11] S. Goldberg, Integrability of almost Kähler manifolds, Proc. Amer. Math. Soc., 21 (1969), 96-100.

[12] L. M. Hervella and A. M. Naveira, Quasi Kähler manifolds, (to appear).

[13] S. Kото̄, Some theorems on almost Kähler spaces, J. Math. Soc. Japan, 12 (1960), 422-433.

[14] A. Newlander and L. Nirenberg, Complex analytic coodinates in almost complex manifolds, Ann. of Math., 65 (1957), 391-404.

[15] G. B. RizzA, Varietá parakähleriane, Annali di Mathematica pura ed applicata (IV), 48 (1974), 47-61.

[16] Y. Tsukaмото, On Kählerian manifolds with positive holomorphic curvature, Proc. Japan Acad., 33 (1957), 333-335.

[17] H. C. WANG, Closed manifolds with homogeneous complex structure, Amer., J. Math., (1954), 1-32,

UNIVERSITÄT ZÜRICH AND UNIVERSITY OF MARYLAND 